\title{
TRACING THE ORIGINS \\ OF SUCCESSFUL AGING: \\ THE ROLE OF ChILDHOOd CONDITIONS \\ AND SOCIETAL CONTEXT
}

Martina Brandt, Christian Deindl,

Karsten Hank

237-2011

( mea-Mannheim Research Institute for the Economics of Aging

L13, 17_D-68131 Mannheim_Phone +49 621 181-2773/1862_Fax +49 621 181-1863_www.mea.uni-mannheim.de 


\title{
Tracing the Origins of Successful Aging: \\ The Role of Childhood Conditions and Societal Context
}

\author{
Martina Brandt ${ }^{\mathrm{a}}$, Christian Deindl ${ }^{\mathrm{b}}$, and Karsten Hank ${ }^{\mathrm{b}}$
}

Abstract: This study investigates the role of childhood conditions and societal context in older Europeans' propensity to age successfully, controlling for later life risk factors. Successful aging was assessed following Rowe and Kahn's conceptualization, using baseline interviews from the first two waves of the Survey of Health, Ageing and Retirement in Europe (SHARE). These data were merged with retrospective life-histories of participants from 13 Continental European countries, collected in 2008-09 as part of the SHARELIFE project. Our sample consists of 22,474 men and women, who are representative of the non-institutionalized population aged 50 or older (mean age: 63.3) in their respective country. Estimating multilevel logistic models, we controlled for demographics (age, sex), childhood conditions (SES, health, cognition), later life risk factors (various dimensions of SES and health behaviors), as well as country-level measures of public social expenditures and social inequality. There is an independent association of childhood living conditions with elders' odds of aging well. Higher parental SES, better math and reading skills, as well as self-reports of good childhood health were positively associated with successful aging, even if contemporary characteristics were controlled for. Later-life SES and health behaviors exhibited the expected correlations with our dependent variable. Moreover, higher levels of public social expenditures and lower levels of income inequality were associated with a greater probability to meet Rowe and Kahn's successful aging criterion. We conclude that unfavorable childhood conditions exhibit a harmful influence on individuals' chances to age well across all European welfare states considered in this study. Policy interventions should thus aim at improving the conditions for successful aging throughout the entire life-course.

Keywords: successful aging; childhood conditions; societal context; Continental Europe; SHARELIFE

\footnotetext{
${ }^{a}$ Corresponding author. MEA - University of Mannheim. Email: brandt@ mea.uni-mannheim.de.

${ }^{\mathrm{b}}$ Institute of Sociology, University of Cologne.
} 


\section{INTRODUCTION}

In the preamble to the Constitution of the World Health Organization (WHO), health is defined as "a state of complete physical, mental and social well-being and not merely the absence of disease or infirmity." Against the background of growing concern about trends in the health of older people in particular (see Crimmins \& Béltran-Sánchez, 2011, for a recent review), Rowe and Kahn (1997; 1998) introduced a highly influential conceptualization of 'successful aging', which adds a social component to merely biomedical conceptualizations of healthy aging. Rowe and Kahn's (1997: 439) definition of successful aging as "avoidance of disease and disability, maintenance of high physical and cognitive function, and sustained engagement in social and productive activities" thus corresponds quite well to WHO's definition of health and has become a commonly applied "gold standard of aging" (Dillaway \& Byrnes, 2009: 706).

Numerous studies showed that current socio-economic status (SES), health behaviors, or religious beliefs, for example, are strong predictors of successful aging (e.g., Crowther et al., 2002; Haveman-Nies et al., 2003; McLaughlin et al., 2010). While these characteristics mainly describe elders' contemporary circumstances, recent research suggested that earlyor midlife factors, such as family background, work characteristics, or the experience of incarceration, matter as well (e.g., Britton et al., 2008; Pruchno et al., 2010). Moreover, a growing body of evidence indicates that childhood SES and health, for example, exhibit long-term influences on individuals' health (e.g, Blackwell et al., 2001; Fors et al., 2009; Luo \& Waite, 2005) and mortality (e.g., Frijters et al., 2010; Hayward \& Gorman, 2004; van den Berg et al., 2009).To our knowledge, though, no research has been conducted yet that explicitly aimed at tracing back the origins of successful aging to individuals' childhood conditions. 
A first objective of the present study, thus, was to explore the potential role of parental SES as well as childhood health and cognition in determining whether individuals succeed in aging well, controlling for an array of contemporary individual characteristics. Data came from the Survey of Health, Ageing and Retirement in Europe (SHARE), including recently collected life-histories of participants aged 50 or over from 13 Continental European countries. Since previous comparative research revealed significant cross-national variation in the prevalence of successful aging (Hank, 2011a), we secondly tested, whether and how societal context contributes to aging well. Previous research suggested a significant role of public social expenditures and social inequality, for example, in shaping opportunities for active and healthy aging (e.g., Hank, 2011b; Wilkinson \& Pickett, 2006). Moreover, current indicators of a country's welfare regime might also reflect, to some degree, macro-level social and economic conditions during individuals' childhood, because the basic set-up of a welfare state ('liberal', 'corporatist', 'social democratic', etc.) is deeply rooted in a country's socio-cultural context and therefore characterized by relative inertia (e.g., Pfau-Effinger, 2005). Along the same lines, Kawachi (2006: 990) suggested that income inequality - as well as social capital - might be considered as "aggregate markers of deeper political and social arrangements (e.g. neoliberalism vs. support for the welfare state, and/or provision of universal primary care services) that are contingent on the history of each country."

\section{METHODS}

Data. - This study uses baseline interviews from the first two waves of the Survey of Health, Ageing and Retirement in Europe (SHARE; cf. Börsch-Supan et al., 2010), which we merged with participants' retrospective life-histories, collected in the survey's third round as part of the SHARELIFE project (see Schröder, 2011, for methodological details). 
Between October 2008 and August 2009 individual life-histories of non-institutionalized respondents aged 50 or older who had already participated in at least one of the previous SHARE waves (conducted in 2004-05 and 2006-07, respectively) were collected in 13 countries: Austria, Belgium, the Czech Republic, Denmark, France, Germany, Greece, Italy, the Netherlands, Poland, Spain, Sweden, and Switzerland. Our analytic sample consists of 22,474 men and women aged 50-96 at baseline (mean: 63.3 years), excluding older adults who required proxy respondents; see Table 1 for descriptive statistics.

Dependent variable. - Following Rowe and Kahn's conceptualization, we defined successful aging as having (a) no major disease, (b) no activity of daily living (ADL) disability, (c) obtaining a median or higher score on tests of cognitive functioning, (d) no more than one difficulty with six measures of physical functioning, and (e) being actively engaged (also see Hank, 2011a; McLaughlin et al., 2010). Accordingly, our dependent variable equals 1 , if all of the above conditions were fulfilled, 0 otherwise. The single items on which this global measure of successful aging is based were operationalized as follows:

(a) Respondents were considered to have no major disease, if they neither reported that a doctor had ever told them they had any of the following chronic diseases: cancer, chronic lung disease, diabetes, heart disease, or stroke, nor obtained a score of four or more on the EURO-D depression scale (see Castro-Costa et al., 2008).

(b) Respondents were classified as having no disability, if they did not report difficulties performing any of the following ADLs: walking across a room, dressing, bathing or showering, eating, getting in or out of bed, and using the toilet.

(c) Participants were considered to have high cognitive functioning if they achieved a median or higher score on a cognitive functioning index based on the following items (see Dewey \& Prince, 2005): naming correctly the day of the week, day, month, and year (1 point for each correct answer: max. 4); an immediate and a delayed 10-word recall test (1 
point for each correctly recalled noun: max. 20); and a mathematical performance test (1 point for each correct answer: max. 5). For missing cognitive items, we computed scores of 0. Participants could obtain a maximum score of 29.

(d) Participants were classified as having high physical functioning if they reported difficulties with at most one of the six following activities: climbing one flight of stairs; climbing several flights of stairs; lifting or carrying items weighing more than $10 \mathrm{lbs}$; stooping, kneeling, or crouching; pulling or pushing large objects; and walking 100 meters.

(e) Respondents were defined as being actively engaged if they reported, first, having done 'any paid work' or 'voluntary or charity work' in the month preceding the interview, or having provided any grandchild care during the past 12 months, and, second, living with a partner, having 'provided help to family, friends, or neighbors' or having 'gone to a sport, social, or other kind of club' in the month preceding the interview.

Contemporary explanatory variables. - We control for two demographic variables, namely sex and age, as well as three measures of the individual's current SES: first, the highest educational degree ever achieved ('low' = lower secondary or second stage of basic education or less; 'medium' = (upper) secondary education or post-secondary non-tertiary education; 'high' = first stage of tertiary education or higher); second, the household equivalent income; and, third, household wealth (in $€$ ). We defined binary indicators of country-specific, purchasing power adjusted income and wealth quartiles, using imputed income and wealth data for respondents with initially missing values (see Christelis et al., 2009, for a description of multiple imputation procedures in SHARE). Moreover, we account for three relevant health behaviors: smoking, frequency of alcohol consumption in the last three months prior to the interview, and regular (i.e. weekly or more often) engagement in vigorous physical activities (such as sports, heavy housework, or a job that involves physical labor). Finally, we include two welfare state related macro-level variables 
in our model, namely the amount of public social expenditures (per capita in US\$; OECD, 2007) and country-specific Gini coefficients (OECD, 2008), indicating the extent of income inequality in a society (cf. Blakely et al., 2002; Hank, 2011b, for similar approaches).

Childhood explanatory variables. - Parental SES when the respondent was 10 years old was measured by (a) the average number of persons sharing a room in the accommodation, and (b) the number of books available in the household (indicated by five categories ranging from 'none or very few (0-10 books)' to 'enough to fill two or more bookcases (more than 200 books)'). After a positive test for linearity, both indicators entered the regression as continuous variables. Cognitive abilities at age 10 were assessed by respondents' self-evaluation of their math and language skills at school in comparison to their classmates (better, same, or worse). Finally, we account for individuals' subjective general health during childhood (five categories ranging from 'excellent' to 'poor'), which entered our model as a continuous variable. Using alternative indicators of childhood health, such as the number of diseases or absence from school due to health problems, did not provide any findings different from those reported below (see Haas \& Bishop, 2010, for an evaluation of the quality of retrospective subjective reports of childhood health).

\section{[Table 1 about here]}

Statistical analysis. - We applied multilevel regression analysis, estimating random intercept models for binary data (e.g., Gelman \& Hill, 2007: Part 2A). In these models, the constant is allowed to vary across countries, that is, it consists of a fixed component and a normally distributed random error term, which takes the same value for all observations within a specific country. This error term measures the deviation of each country from the fixed part of the constant, thereby accounting for the correlation between individuals nested within the same country and capturing otherwise unobserved context effects. If the variance 
of the macro-level error term turns out to be statistically significant from zero, such effects are present. - The results of the logistic regressions are presented as odds ratios (OR).

\section{RESULTS}

The explanatory variables were included stepwise into the regression, that is, we started with a so called 'empty' model that contained only the constant and the macro-level error term (Model 1). The contemporary (i.e., later-life) micro-level control variables were introduced in Model 2, which was complemented by our set of childhood variables in Model 3. Finally, we added our two macro-level variables in Model 4 (see Table 2). - Note that all findings reported here are based on the pooled SHARE sample. In addition, we conducted separate analyses for men and women, as well as for different cohorts. These supplementary analyses did not provide any further insights, though (results are available from the authors upon request).

We begin our description of results by examining the outcomes of the contemporary micro-level control variables (Model 2). Looking at individuals' basic demographic characteristics shows that the risk of failing to meet Rowe and Kahn's successful aging criterion sharply increases with age and is significantly higher among women than men $(\mathrm{OR}=0.65,95 \% \mathrm{CI}=0.609-0.700)$. We also find the expected positive socio-economic gradient: the chances of aging well increase steadily with individuals' educational attainment and across all income and wealth quartiles. Finally, health behaviors are shown to matter greatly: while former and current smokers as well as respondents who are not physically active exhibit the lowest odds, individuals reporting to consume alcohol at least occasionally are most likely to age successfully (cf. Britton et al., 2008).

The inclusion of childhood variables (Model 3) barely changes the coefficients of the contemporary controls, but significantly improves the model fit (LR-test: $90.73^{* * *}$ ). Both 
measures of parental SES suggest that individuals who experienced a higher socioeconomic position at age 10 have a higher propensity to age well than their less advantaged counterparts (number of persons per room: $\mathrm{OR}=0.92,95 \% \mathrm{CI}=0.890-0.950$; number of books in household: $\mathrm{OR}=1.06,95 \% \mathrm{CI}=1.024-1.088)$. Individuals reporting below average cognitive skills (in terms of math and/or language proficiency) at age 10 are significantly more likely to fail the successful aging criterion, as are those reporting poorer levels of general health during their childhood $(\mathrm{OR}=0.86,95 \% \mathrm{CI}=0.836-0.895)$.

Finally, we turn to cross-country differences in and societal determinants of successful aging. Consistent with descriptive findings from previous research (Hank, 2011a), the 'empty' Model 1 clearly indicates a statistically significant regional variation of the constant, with an intra-class correlation (ICC) of $4 \%$ (which is an order of magnitude we also find in similar studies; see, for example, Hank, 2011b). The ICC increases to 7\% and 9\%, respectively, if we control for contemporary (Model 2) and childhood (Model 3) individual characteristics. This indicates that actual country differences in successful aging are substantially underestimated, if one does not account for differences in population composition (see Gelman \& Hill, 2007: Section 21.7). If, however, we control for social expenditures and social inequality (in Model 4), the ICC is reduced to 3\%, that is, one third of its size in Model 3. The inclusion of these variables also further improves the model fit (LR-test: 17.81***) and the coefficients of both macro-indicators are statistically significant, suggesting that individuals living in countries with higher levels of public social expenditures are more likely to succeed in aging well $(\mathrm{OR}=1.04,95 \% \mathrm{CI}=1.008-1.079)$, whereas those living in a society characterized by greater levels of income inequality are less likely to enter old age successfully $(\mathrm{OR}=0.95,95 \% \mathrm{CI}=0.937-0.967)$.

[Table 2 about here] 


\section{DISCUSSION}

A primary objective of the present study was to explore the potential role of parental SES as well as childhood health and cognition in determining whether older Europeans succeed in aging well. Using new retrospective life-history data from the SHARELIFE project, we found an independent association of childhood living conditions with elders' probability to meet the successful aging criteria suggested by Rowe and Kahn $(1997 ; 1998)$. Higher parental SES, better math and reading skills, as well as self-reports of good childhood health were positively associated with successful aging, even if an array of contemporary characteristics was controlled for. Accounting for childhood conditions significantly improved the model fit compared to a model that included individuals' contemporary characteristics only. We did not detect significant differences between the importance of these predictors in men and women, or in different cohorts (details not shown).

The correlations of later-life SES and health behaviors with our dependent variable confirmed previous findings from Anglo-Saxon countries (e.g., Britton et al., 2008; McLaughlin et al., 2010) for a relatively broad set of 13 Continental European countries. Against the background of widely varying proportions of successfully aging elders in these countries (cf. Hank, 2011a) and exploiting the advantage of having a cross-nationally comparative data set at our disposal, we also investigated whether and how societal context contributes to aging well. The results of our multilevel analysis clearly indicate a significant role of public social expenditures and social inequality in individuals' odds to meet Rowe and Kahn's successful aging criterion (see Hank, 2011b; Wilkinson \& Pickett, 2006, for a detailed discussion of potential underlying mechanisms). Including these macro-level welfare indicators also contributed to explaining the cross-national variation in proportions of elders aging well. 
The policy implications of these findings are clear: welfare states do play an important role in establishing opportunity structures promoting successful aging - and should act accordingly. Policy interventions should aim at improving conditions for successful aging throughout the entire life-course, starting in childhood and providing individuals with (ideally) equal opportunities for education and health in particular. Along these lines, the European Commission has established programs for lifelong learning (e.g., Commission of the European Communities, 2000) or healthy aging (e.g., Jamieson, 1994). In order to be effective, however, it is important that such programs' initial intervention takes place early in the individual's life-course. Erlinghagen (2010), for example, showed that the experience of volunteering in mid-life plays a major role in retirees' decision to volunteer. Moreover, attention needs to be paid to the interrelation between different dimensions of successful aging (see, for example, Sirven \& Debrand, 2008, whose findings suggest a positive impact of active on healthy aging), that is, one needs to take a 'holistic' perspective.

This latter issue points to a potential limitation which our research shares with many other studies on successful aging: is our observed outcome really an appropriate measure of aging well (e.g., Dillaway \& Byrnes, 2009)? Research exploring self-ratings and lay views of successful aging regularly documented greater diversity and more domains than those accounted for in academic conceptualizations (e.g., Hung et al., 2010; Strawbridge et al., 2002). Moreover, specific domains of successful aging might be valued differently by older people across cultures (e.g., Fernández-Ballesteros et al., 2008; Hung et al., 2010), whereas we assume that Rowe and Kahn's concept measures successful aging in a comparable way across Continental Europe. Particularly in case of the 'active engagement' criterion, the items which are most relevant to constitute an individual's classification as being 'successful' might be contextually bound (e.g., Meijs et al., 2003), and it is also well- 
known that self-reported health measures might vary cross-nationally simply due to reporting or diagnostic differences (e.g., Jürges, 2007). These potential limitations provide no argument, however, that might corrupt our overall conclusion of a long-standing impact of childhood conditions and welfare state arrangements on individuals' chances to age successfully.

\section{ACKNOWLEDGEMENTS}

This paper uses data from SHARELIFE release 1, as of November 24th 2010 and from SHARE release 2.3.1, as of July 29th 2010. The SHARE data collection has been primarily funded by the European Commission through the 5th framework program (project QLK6-CT-2001- 00360 in the thematic program Quality of Life), through the 6th framework program (projects SHARE-I3, RII-CT- 2006-062193, COMPARE, CIT5-CT2005-028857, and SHARELIFE, CIT4-CT-2006-028812) and through the 7th framework program (SHARE-PREP, 211909 and SHARE-LEAP, 227822). Additional funding from the U.S. National Institute on Aging (U01 AG09740-13S2, P01 AG005842, P01 AG08291, P30 AG12815, Y1-AG-4553-01 and OGHA 04-064, IAG BSR06-11, R21 AG025169) as well as from various national sources is gratefully acknowledged (see www.shareproject.org/t3/share/index.php for a full list of funding institutions).

\section{REFERENCES}

Blackwell, D.L., Hayward, M.D., \& Crimmins, E.M. (2001). Does childhood health affect chronic morbidity in later life? Social Science \& Medicine, 52, 1269-1284.

Blakely, T.A., Lochner, K., \& Kawachi, I. (2002). Metropolitan area income inequality and self-rated health - a multilevel study. Social Science \& Medicine, 54, 65-77. 
Börsch-Supan, A., Hank, K., Jürges, H., \& Schröder, M. (2010): Longitudinal Data Collection in Continental Europe: Experiences from the Survey of Health, Ageing and Retirement in Europe. In J.A. Harkness et al. (Eds.), Survey Methods in Multicultural, Multinational, and Multiregional Contexts (pp. 507-514). Hoboken, NJ: John Wiley \& Sons.

Britton, A., Shipley, M., Singh-Mannoux, A., \& Marmot, M.G. (2008). Successful aging: The contribution of early-life and midlife risk factors. Journal of the American Geriatrics Society, 56, 1098-1105.

Castro-Costa, E., Dewey, M., Stewart, R., Banerjee, S., Huppert, F., Medonca-Lima, C., Bula, C., Reisches, F., Wancata, J., Ritchie, K., et al. (2008). Ascertaining late-life depressive symptoms in Europe: an evaluation of the survey version of the EURO-D scale in 10 nations. The SHARE project. International Journal of Methods in Psychiatric Research, 17, 12-29.

Commission of the European Communities (2000). A memorandum on lifelong learning (Commission Staff Working Paper), Brussels.

Crimmins, E.M. \& Béltran-Sánchez, H. (2011). Mortality and morbidity trends: is there compression of morbidity? Journal of Gerontology: Social Sciences, 66B, 75-86.

Christelis, D., Japelli, T., Paccagnella, O., \& Weber, G. (2009). Income, wealth, and financial fragility in Europe. Journal of European Social Policy, 19, 359-376.

Crowther, M.R., Parker, M.W., Achenbaum, W.A., Larimore, W.L., \& Koenig, H.G. (2002). Rowe and Kahn's model of successful aging re-visited: Positive spirituality the forgotten factor. The Gerontologist, 42, 613-620.

Dewey, M. \& Prince, M. (2005). Cognitive function. In A. Börsch-Supan et al. (Eds.), Health, Ageing and Retirement in Europe - First results from SHARE (pp. 118-125). Mannheim: MEA. 
Dillaway, H.E. \& Byrnes, M. (2009). Reconsidering successful aging. A call for renewed and expanded academic critiques and conceptualizations. Journal of Applied Gerontology, 28, 702-722.

Erlinghagen, M. (2010). Volunteering after retirement. Evidence from German panel data. European Societies, 12, 603-625.

Fernández-Ballesteros, R., Garcıá, L.F., Abarca, A., Blanc, L., Efklide, A., Kornfeld, R., Lerma, A.J., Mendoza-Nunez, V., Mendoza-Ruvalcaba, N.M., Orosa, T., et al. (2008). Lay concept of ageing well: cross-cultural comparisons. Journal of the American Geriatrics Society, 56, 950-952.

Fors, S., Lennartsson, C., \& Lundberg, O (2009). Childhood living conditions, socioeconomic position in adulthood, and cognition in later life: Exploring the associations. Journal of Gerontology: Social Sciences, 64B, 750-757.

Frijters, P., Hatton, T.J., Martin, R.M., \& Shileds, M.A. (2010). Childhood economic conditions and length of life: Evidence from the UK Boyd Orr cohort, 1937-2005. Journal of Health Economics, 29, 39-47.

Gelman, A., \& Hill, J. (2007). Data Analysis Using Regression and Multilevel / Hierarchical Models. Cambridge University Press: Cambridge.

Hank, K. (2011a). How "Successful” Do Older Europeans Age? Findings From SHARE. Journal of Gerontology: Social Sciences [online advance access].

Hank, K. (2011b). Societal determinants of productive aging: A multilevel analysis across 11 European countries. European Sociological Review [online advance access].

Haas, S.A. \& Bishop, N.J. (2010). What do retrospective subjective reports of childhood health capture? Evidence from the Wisconsin Longitudinal Study. Research on Aging, 32, 698-714. 
Haveman-Nies, A., de Groot, L., \& van Staveren, W.A. (2003). Dietary quality, lifestyle factors and healthy ageing in Europe: the SENECA study. Age and Ageing, 32, 427434.

Hayward, M.D. \& Gorman, B.K. (2004). The long arm of childhood: The influence of early-life social conditions on men's mortality. Demography, 41, 87-107.

Hung, L.-W., Kempen, G.I.J.M., \& De Vries, N.K. (2010). Cross-cultural comparisons between academic and lay views of healthy ageing: a literature review. Ageing \& Society, 30, 1373-1391.

Jamieson, A. (1994). The role of the European Union in promoting healthy ageing. Social Science \& Medicine, 39, 1497-1499.

Jürges, H. (2007): True health vs. response styles: exploring cross-country differences in self-reported health. Health Economics, 16, 163-178.

Kawachi, I. (2006). Commentary: Social capital and health: making the connections one step at a time. International Journal of Epidemiology, 35, 989-993.

Luo, Y. \& Waite, L.J. (2005). The impact of childhood and adult SES on physical, mental, and cognitive well-being in later-life. Journal of Gerontology: Social Sciences, 60B, S93-S101.

McLaughlin, S.J., Connell, C.M., Heeringa, S.G., Li, L.W., \& Roberts, J.S. (2010). Successful aging in the United States: prevalence estimates from a national sample of older adults. Journal of Gerontology: Social Sciences, 65B, 216-226.

Meijs, L.C.P.M., Handy, F., Cnaan, R.A., Brudney, J.L., Ascoli, U., Ranade, S., Hustinx, L., Weber, S., \& Weiss, I. (2003). All in the eyes of the beholder? Perceptions of volunteering across eight countries. In P. Dekker \& L. Halman (Eds.), The Values of Volunteering. Cross-Cultural Perspectives (pp. 19-34). New York (et al.): Kluwer Academic / Plenum Publishers. 
OECD (2007). OECD Social Expenditure Data Base (SOCX). Paris: OECD.

OECD (2008). Growing Unequal? Income Distribution and Poverty in OECD Countries. Paris: OECD.

Pfau-Effinger, B. (2005). Culture and Welfare State Policies: Reflections on a Complex Interaction. Journal of Social Policy, 34, 3-20.

Pruchno, R.A., Wilson-Genderson, M., Rose, M., \& Cartwright, F. (2010). Successful aging: Early influences and contemporary characteristics. The Gerontologist, 50, 821833.

Sirven, N. \& Debrand, T. (2008). Social participation and healthy ageing: an international comparison using SHARE data. Social Science \& Medicine, 67, 2017-2026.

Strawbridge, W.J., Wallhagen, M.I., \& Cohen, R.D. (2002). Successful aging and wellbeing. Self-rated compared with Rowe and Kahn. The Gerontologist, 42, 727-733.

Rowe, J.W. \& Kahn, R.L. (1997). Successful aging. The Gerontologist, 37, 433-440.

Rowe, J.W. \& Kahn, R.L. (1998). Successful aging. New York: Pantheon Books.

Schröder, M. (Ed.) (2011). Retrospective Data Collection in the Survey of Health, Ageing and Retirement in Europe. SHARELIFE Methodology. Mannheim: MEA.

van den Berg, G., Doblhammer, G., \& Christensen, K. (2009). Exogenous determinants of early-life conditions, and mortality later in life. Social Science \& Medicine, 68, 15911598.

Wilkinson, R.G. \& Pickett, K.E. (2006). Income inequality and population health: A review and explanation of the evidence. Social Science \& Medicine, 62, 1768-1784. 
TABLES

Table 1: Pooled sample characteristics (unweighted)

Successful aging $28 \%$

\section{Contemporary individual controls}

Demographics

Age

$-\quad 50-59$

$41 \%$

- $60-69$

$33 \%$

- 70 or older

$26 \%$

Sex: female

$55 \%$

SES

Education

- Low

$47 \%$

- $\quad$ Medium

$33 \%$

- High

$20 \%$

Income (mean)

$21,461 €$

Wealth (mean)

$241,805 €$

Health behaviors

Smoking

- Never

$52 \%$

- Stopped

$28 \%$

- Current

$21 \%$

Alcohol consumption in last three months

- Never

$36 \%$

- Twice a month or less often

$12 \%$

- At least once a week

$27 \%$

- Almost every day

$25 \%$ 


\section{Childhood conditions}

SES

- No. of individuals per room

- No. of books in household
0-10 books
$43 \%$
11-25 books
$23 \%$
26-100 books
$21 \%$
101-200 books
$6 \%$
More than 200 books
$6 \%$

Cognition - math skills

- Worse than others

- Same as others

$51 \%$

- Better than others

$35 \%$

Cognition - language skills

- Worse than others

- Same as others

$50 \%$

- Better than others

$37 \%$

Childhood health ('excellent' $\rightarrow$ 'poor')

- Excellent

- Very good

- Good

- Fair

- Poor
$36 \%$

$34 \%$

$23 \%$

$6 \%$

$2 \%$

\section{Macro-level indicators}

Public social expenditures (1,000 US\$ per capita) 6.9

Gini coefficient

30.2

Source: SHARE (Waves 1-3); OECD (2007, 2008); n=22,474 individuals; 13 countries. 
Table 2: Results of multilevel logistic regressions for 'successful aging' - odds ratios (95\% confidence intervals)

\begin{tabular}{|c|c|c|c|c|c|}
\hline & & Model (1) & Model (2) & Model (3) & Model (4) \\
\hline \multicolumn{6}{|c|}{ Contemporary individual controls } \\
\hline \multicolumn{6}{|c|}{ Demographics } \\
\hline \multicolumn{6}{|c|}{ Age } \\
\hline- & $50-59^{\mathrm{a}}$ & & 1.00 & 1.00 & 1.00 \\
\hline \multirow{2}{*}{\multicolumn{2}{|c|}{$-\quad 60-69$}} & & $0.50 * *$ & $0.51 * *$ & $0.50 * *$ \\
\hline & & & $(0.469-0.542)$ & $(0.474-0.549)$ & $(0.463-0.537)$ \\
\hline \multirow{2}{*}{\multicolumn{2}{|c|}{ - $\quad 70$ or older }} & & $0.17 * *$ & $0.17 * *$ & $0.16^{* *}$ \\
\hline & & & $(0.151-0.187)$ & $(0.150-0.187)$ & $(0.145-0.181)$ \\
\hline \multirow{2}{*}{\multicolumn{2}{|c|}{ Sex: female }} & & $0.65 * *$ & $0.64 * *$ & $0.65 * *$ \\
\hline & & & $(0.609-0.700)$ & $(0.595-0.687)$ & $(0.601-0.693)$ \\
\hline \multicolumn{6}{|c|}{ SES } \\
\hline \multicolumn{6}{|c|}{ Education } \\
\hline- & Low $^{\mathrm{a}}$ & & 1.00 & 1.00 & 1.00 \\
\hline \multirow{2}{*}{\multicolumn{2}{|c|}{ - $\quad$ Medium }} & & $1.56^{* *}$ & $1.38 * *$ & $1.37 * *$ \\
\hline & & & $(1.448-1.688)$ & $(1.271-1.492)$ & $(1.261-1.481)$ \\
\hline \multirow{2}{*}{\multicolumn{2}{|c|}{ - High }} & & $2.03 * *$ & $1.66^{* *}$ & $1.68 * *$ \\
\hline & & & $(1.856-2.217)$ & $(1.501-1.830)$ & $(1.524-1.861)$ \\
\hline \multicolumn{6}{|c|}{ Income } \\
\hline & $1^{\text {st }}$ quartile ${ }^{\mathrm{a}}$ & & 1.00 & 1.00 & 1.00 \\
\hline \multirow{2}{*}{\multicolumn{2}{|c|}{ - $\quad 2^{\text {nd }}$ quartile }} & & $1.16^{* *}$ & $1.16^{* *}$ & $1.17 * *$ \\
\hline & & & $(1.050-1.288)$ & $(1.047-1.285)$ & $(1.054-1.294)$ \\
\hline \multirow{2}{*}{\multicolumn{2}{|c|}{ - $\quad 3^{\text {rd }}$ quartile }} & & $1.51 * *$ & $1.49 * *$ & $1.50 * *$ \\
\hline & & & $(1.369-1.665)$ & $(1.349-1.643)$ & $(1.355-1.650)$ \\
\hline \multirow{2}{*}{\multicolumn{2}{|c|}{ - $\quad 4^{\text {th }}$ quartile }} & & $1.72 * *$ & $1.69 * *$ & $1.69 * *$ \\
\hline & & & $(1.561-1.904)$ & $(1.527-1.865)$ & $(1.534-1.873)$ \\
\hline \multicolumn{6}{|c|}{ Wealth } \\
\hline- & $1^{\text {st }}$ quartile $^{\mathrm{a}}$ & & 1.00 & 1.00 & 1.00 \\
\hline \multirow{2}{*}{\multicolumn{2}{|c|}{ - $\quad 2^{\text {nd }}$ quartile }} & & $1.40 * *$ & $1.36^{* *}$ & $1.36^{* *}$ \\
\hline & & & $(1.268-1.553)$ & $(1.226-1.504)$ & $(1.232-1.511)$ \\
\hline \multirow{2}{*}{\multicolumn{2}{|c|}{$-\quad 3^{\text {rd }}$ quartile }} & & $1.64 * *$ & $1.58 * *$ & $1.59 * *$ \\
\hline & & & $(1.481-1.808)$ & $(1.425-1.742)$ & $(1.439-1.759)$ \\
\hline \multirow{2}{*}{\multicolumn{2}{|c|}{ - $\quad 4^{\text {th }}$ quartile }} & & $1.83 * *$ & $1.73 * *$ & $1.75^{* *}$ \\
\hline & & & $(1.655-2.023)$ & $(1.563-1.914)$ & $(1.580-1.935)$ \\
\hline \multicolumn{6}{|c|}{ Health behaviors } \\
\hline \multicolumn{6}{|c|}{ Smoking } \\
\hline- & Never $^{\mathrm{a}}$ & & 1.00 & 1.00 & 1.00 \\
\hline \multirow{2}{*}{\multicolumn{2}{|c|}{ - $\quad$ Stopped }} & & $0.80 * *$ & $0.81 * *$ & $0.82 * *$ \\
\hline & & & $(0.743-0.872)$ & $(0.751-0.882)$ & $(0.756-0.888)$ \\
\hline- & Current & & $0.81 * *$ & $0.81 * *$ & $0.82 * *$ \\
\hline & & & $(0.747-0.888)$ & $(0.741-0.881)$ & $(0.753-0.898)$ \\
\hline Alcoh & consumption ( 3 months) & & & & \\
\hline- & Never $^{\mathrm{a}}$ & & 1.00 & 1.00 & 1.00 \\
\hline- & Twice a month or less often & & $1.45 * *$ & $1.44 * *$ & $1.41 * *$ \\
\hline & & & $(1.297-1.615)$ & $(1.292-1.610)$ & $(1.264-1.577)$ \\
\hline- & At least once a week & & $1.60 * *$ & $1.54 * *$ & $1.51 * *$ \\
\hline & & & $(1.466-1.753)$ & $(1.409-1.689)$ & $(1.378-1.655)$ \\
\hline- & Almost every day & & $1.38 * *$ & $1.28 * *$ & $1.33 * *$ \\
\hline & & & $(1.254-1.515)$ & $(1.168-1.414)$ & $(1.209-1.468)$ \\
\hline Vigor & s physical activities & & $1.70 * *$ & $1.68 * *$ & $1.67 * *$ \\
\hline & & & $(1.585-1.815)$ & $(1.568-1.798)$ & $(1.564-1.793)$ \\
\hline
\end{tabular}


Table 2 (cont'd.): Results of multilevel logistic regressions for 'successful aging' - odds ratios (95\% confidence intervals)

\section{Childhood conditions}

Model (1) $\quad$ Model (2) $\quad$ Model (3) $\quad$ Model (4)

\section{SES}

No. individuals per room

No. of books in household

Cognition - math skills

- Worse than others

- $\quad$ Same as others ${ }^{\mathrm{a}}$

- Better than others

Cognition - language skills

- Worse than others

- Same as others ${ }^{\mathrm{a}}$

- $\quad$ Better than others

Childhood health ('excellent' $\rightarrow$ 'poor')

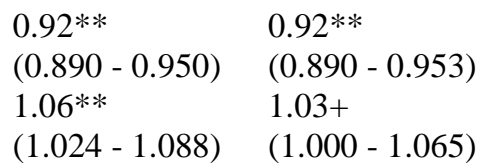

Macro-level indicators

Social expenditures (per capita)

$1.04 *$

$(1.008-1.079)$ $0.95 * *$ (0.937 - 0.967)

BIC 25741.88

22046.78

21935.47

21695.32

LL $-12860.92$ $-10928.2$

0.13

0.24 $-10837.47$ $-10819.66$

Variance (country)

0.01

0.02

0.04

0.07

0.34

0.10

ICC

No. of observations

Persons

Countries 13

Source: SHARE (Waves 1-3). Significance: $* * \mathrm{p}<0.01, * \mathrm{p}<0.05,+\mathrm{p}<0.1{ }^{\mathrm{a}}{ }^{\mathrm{a}}$ Reference category.

LR-test (2) vs (1): 1932.72***, df 17; LR-test (3) vs (2): 90.73***, df 7; LR-test (4) vs (3): $17.81 * * *$, df 2 